\title{
PERFIL DOS UNIVERSITÁRIOS DE ODONTOLOGIA E O IMPACTO DA SAÚDE BUCAL NA QUALIDADE DE VIDA DESSA POPULAÇÃO
}

\author{
Tatiana Paiva Moreira FRANCO' \\ Vicente Canuto da MOTTA ${ }^{1}$ \\ Antonio Canabarro ${ }^{2}$ \\ Patricia Nivoloni TANNURE² \\ E-mail pntannure@gmail.com
}

\section{RESUMO}

Objetivou-se identificar as condições de saúde bucal de universitários do curso de odontologia de uma instituição de ensino particular e avaliar o impacto da saúde bucal na qualidade de vida dessa população. A pesquisa foi realizada durante o período de 8 meses e foi composta por universitários de odontologia com interesse em realizar clareamento dentário caseiro. Na anamnese dados sociais, história médica e odontológica, queixa principal, uso de substâncias corantes, tratamento ortodôntico realizado e presença de sensibilidade dentária foram coletados. Ao exame clínico intrabucal observou-se retração gengival, biofilme visível, índice de cárie e fluorose. Os graduandos responderam a um questionário para mensurar o impacto da saúde bucal na qualidade de vida (OHIP14). Quarenta e sete graduandos do curso de odontologia com média de idade de 23,5 anos (dp 5,5) que passaram a infância na capital do Rio de Janeiro $(72,5 \%)$ e que não apresentavam doenças sistêmicas $(87,2 \%)$ foram incluídos na amostra. A grande maioria (66\%) realizou tratamento ortodôntico, 14,9\% apresentavam retração gengival e 36,2\% apresentavam biofilme visível no momento do estudo. Uma grande parte da população $(44,7 \%)$ apresentava dentição hígida e o CPOD foi de $4,13( \pm 4,5)$. A fluorose leve ou muito leve afetou $6,4 \%$ dos participantes. Observou-se ainda uma boa qualidade de vida $(\mathrm{OHIP}=4,9( \pm 5,6))$ sem impacto negativo relacionado à saúde bucal. Não houve associação positiva entre o gênero e a dentição hígida e entre biofilme visível e dentição hígida $(p>0,05)$. Foi observada uma associação significativa entre a ausência de tratamento ortodôntico e dentição hígida $(p=0,01)$. Pode-se concluir que, em um número expressivo, os universitários participantes se encontravam saudáveis e com a dentição hígida, entretanto, estavam insatisfeitos em relação à forma, posição e cor dos dentes. Não foi observado impacto negativo na qualidade de vida relacionada à saúde bucal.

DESCRITORES: SERVIÇOS DE SAÚDE PARA ESTUDANTES; ESTUDANTES DE ODONTOLOGIA; SAÚDE BUCAL 
FRANCO TPM, MOTTA VC, CANABARRO A, TANNURE PN. Perfil dos universitários de odontologia e o impacto da saúde bucal na qualidade de vida dessa população. Rev. Odontol. Univ. Cid. São Paulo 2018 jul/set 30(3) 256-264

ISSN 1983-5183

\title{
PROFILE OF UNIVERSITY STUDENTS IN DENTISTRY AND THE ORAL HEALTH IMPACT ON THEIR QUALITY OF LIFE
}

\begin{abstract}
The aim of this study was to identify the dental health conditions of students in the dental school of a private educational institution and to evaluate the impact of oral health on the quality of life of this population. The research was carried out during 8 months and was composed by students of dentistry with interest in dental bleaching at home. During the anamnesis, social data, medical and dental history, main complaint, use of coloring substances, orthodontic treatment performed and tooth sensitivity were collected. The intra-oral clinical examination revealed gingival retraction, visible biofilm, caries index and fluorosis. Graduates responded to a questionnaire to measure the impact of oral health on quality of life (OHIP14). Forty-seven graduates of the dentistry course with a mean age of 23.5 years (SD 5.5) who spent their childhood in the capital city of Rio de Janeiro (72.5\%) and did not present systemic diseases (87.2\%) were included in the sample. The majority (66\%) underwent orthodontic treatment, $14.9 \%$ had gingival retraction, and $36.2 \%$ presented visible biofilm at the time of the study. A large part of the population (44.7\%) had a healthy dentition and the DMFT was $4.13( \pm 4.5)$. The mild or very mild fluorosis affected $6.4 \%$ of the participants. There was also a good quality of life $(\mathrm{OHIP}=4.9( \pm 5.6))$ with no negative impact related to oral health. There was no positive association between gender and healthy dentition and between visible biofilm and healthy dentition ( $p>0.05$ ). A significant association between absence of orthodontic treatment and healthy dentition was observed $(p=0.01)$. It can be concluded that an expressive number of students were healthy, however, they were not satisfied with the shape, position and color of their teeth. No negative impact on oral health related quality of life was observed.
\end{abstract}

DESRIPTORS: STUDENT HEALTH SERVICES; STUDENTS, DENTAL; ORAL HEALTH

\section{INTRODUÇÃO}

A saúde da população de estudantes universitários deve estar entre as prioridades das instituições de Ensino Superior. A proposta dos projetos de Universidades Saudáveis, que têm recebido crescente interesse, é encorajar o desenvolvimento de determinados aspectos, tais como a criação de ambientes de trabalho, a aprendizagem e a convivência saudáveis, proteger o meio ambiente e promover práticas promotoras de saúde ${ }^{1,2}$. Além disso, as universidades formam futuros profissionais e formuladores de políticas que poderão influenciar as condições de saúde e qualidade de vida de outras pessoas 3 .

Na sociedade atual é crescente o interesse e a busca por uma melhor qualidade de vida (QV). Ao abordar aspectos ligados à QV são também apurados os hábitos que compõem o estilo de vida de um indivíduo. Sabe-se que os hábitos adotados durante a vida, tanto em relação à escolha nutricional e à prática de atividades físicas, quanto em relação à utilização de drogas ilícitas e consumo de álcool e tabaco, estão diretamente relacionados ao contexto de influência na QV4.

O tratamento odontológico pode gerar modificações na melhoria da saúde oral e geral, na autopercepção de saúde e na adesão dos pacientes aos serviços de saúde, influenciando significativamente na QV55. Esse conceito relacionado com a saúde bucal é multidimensional e inclui a avaliação subjetiva da saúde bucal do indivíduo, aspectos funcionais, bem-estar geral e bem-estar emocional, expectativas e satisfação com o seu autocuidado ${ }^{6}$. 
FRANCO TPM, MOTTA VC, CANABARRO A, TANNURE PN. Perfil dos universitários de odontologia e o impacto da saúde bucal na qualidade de vida dessa população. Rev. Odontol. Univ. Cid. São Paulo 2018 jul/set 30(3) 256-264

ISSN 1983-5183

Em relação à saúde bucal, apesar dos inúmeros benefícios do flúor na doença cárie, altas concentrações no organismo desse íon durante o desenvolvimento do germe dentário podem levar à fluorose. Decorrente da ingestão excessiva e crônica de flúor e caracterizada por manchas brancas, opacas e sem brilho, estriadas e hipoplásicas, presentes em dentes homólogos, sua prevalência tem aumentado na população brasileira. A gravidade da fluorose varia com a susceptibilidade individual e o grau de exposição, dose e duração7. Os resultados do último levantamento epidemiológico no Brasil relataram que a prevalência de fluorose dentária em crianças de 12 anos de idade foi de 16,7\% e 15,1\% foram representados pelos níveis de severidade muito leve e leve. A maior prevalência de crianças com fluorose foi observada na Região Sudeste $(19,1 \%)^{8}$.

Em relação à cárie, tem-se observado o seu declínio no Brasil e no mundo. Entretanto, sua prevalência ainda é alta em determinados grupos populacionais e se mantém como um problema de saúde pública neste país ${ }^{9}$. De acordo com os resultados provenientes da Pesquisa Nacional de Saúde Bucal - 2010, entre os adultos, a necessidade de algum tipo de prótese dentária ocorre em $69 \%$ dos $\operatorname{casos}^{8}$. Na maioria dos casos a necessidade de prótese dentária está associada a dentes com lesões de cárie não tratada que evoluíram para a exodontia do elemento dentário.

Diante da necessidade de se avaliar a saúde dos jovens estudantes do ensino superior e da importância da mesma perante o desenvolvimento dessa população, torna-se interessante investigar o perfil e as condições dentárias dos universitários e o impacto na sua qualidade de vida. O objetivo deste estudo foi identificar o perfil e as condições de saúde bucal de universitários do curso de odontologia de uma instituição de ensino particular e avaliar o impacto da saúde bucal na qualidade de vida dessa população.

\section{MATERIAIS E MÉTODOS}

Este estudo foi submetido à avaliação e aprovação pelo Comitê de Ética em pesquisa da Universidade Veiga de Almeida (2.213.422).

A pesquisa foi realizada na Clínica do Mestrado Profissional em Odontologia e a amostra de conveniência foi composta por universitários de odontologia com interesse em realizar clareamento dentário caseiro. Os atendimentos clínicos ocorreram uma vez por mês durante 8 meses do ano de 2016. Todos os participantes assinaram o Termo de Consentimento Livre e Esclarecido. Durante a anamnese os dados coletados através do prontuário odontológico foram os seguintes: ano do curso, sexo, idade, local de infância, história médica pregressa, uso de medicamentos, fumo, queixa principal, uso de substâncias corantes, tratamento ortodôntico realizado e presença de sensibilidade dentária. Ao exame clínico intrabucal foi observada a presença de retração gengival com sonda periodontal milimetrada tipo Carolina do Norte (Trinity ${ }^{\circledast}$ - São Paulo, SP, Brasil), medida da junção cemento-esmalte (JCE) até a margem gengival (MG). Para o dado biofilme visível utilizou-se o índice dicotômico conferindo 1 para a presença de biofilme e 0 para a ausência. Todos os dentes foram avaliados, sendo 4 sítios por dente, exceto os terceiros molares. A presença de retração gengival foi registrada se pelo menos 1 sítio com distância JCE-MG $\geq 1 \mathrm{~mm}$ fosse detectado. Foi coletado ainda o índice CPOD segundo os critérios da OMS e o Índice de Dean (Dean, 1934) que classifica a fluorose em seis níveis segundo os aspectos clínicos: normal, questionável, muito leve, leve, moderado e severo. Os graduandos responderam a um questionário (OHIP-14) para mensurar o impacto da saúde bucal na qualidade de vida. Para calcular o impacto da saúde bucal na qualidade de vida (ISBQV) através do OHIP-14, utilizou-se o chamado "método aditivo" no qual as 14 respostas foram codificadas segundo uma escala de 5 pontos, variando de 0 para "nunca" até 4 para "sempre" e todos os valores atribuídos às respostas foram somados. Dessa forma, tais pontuações poderiam variar de 0 a $56^{10,11,12}$ e, quanto maior a 
FRANCO TPM, MOTTA VC, CANABARRO A, TANNURE PN. Perfil dos universitários de odontologia e o impacto da saúde bucal na qualidade de vida dessa população. Rev. Odontol. Univ. Cid. São Paulo 2018 jul/set 30(3) 256-264

ISSN 1983-5183

pontuação, maior a percepção do impacto da saúde pelos examinados. Todos os dados foram tabulados e armazenados no programa SPSS 22.0 e a análise estatística foi realizada através dos testes Qui-quadrado usando-se como nível de significância o valor de $p<=0,05$.

\section{RESULTADOS}

A amostra deste estudo foi composta por 47 graduandos do curso de Odontologia da Universidade Veiga de Almeida com média de idade de 23,5 anos $( \pm 5,5)$. Na sua grande maioria eram mulheres $(n=40)$ que haviam passado sua infância na capital do Rio de Janeiro $(72,5 \%)$ e que não apresentavam doenças sistêmicas (87,2\%). Todos os universitários declararam não fazer uso de tabaco. A maior parte $(53,0 \%)$ dessa população declarou alimentar-se com substâncias corantes (café, mate, refrigerante) e os principais fatores que causavam incômodo nessa população foram a cor e a forma ou a posição dos dentes (31,9\% e 25,5\%) respectivamente. Na Tabela 1 encontram-se informações sobre as características dessa população, dados sobre a saúde geral e queixa principal. Na Tabela 2 são apresentadas informações sobre a história dental passada e atual da população estudada. A grande maioria (66\%) realizou tratamento ortodôntico, porém 55,3\% o finalizaram. Apenas 6,4\% relataram sentir sensibilidade dentária. Dentre os dados sobre as condições saúde bucal, observou-se que 14,9\% apresentavam retração gengival e 36,2\% apresentavam biofilme visível no momento do estudo. Uma grande parte da população $(44,7 \%)$ apresentava dentição hígida, sendo que $72,3 \%$ tinham os dentes anteriores hígidos. A fluorose leve ou muito leve afetou $6,4 \%$ dos participantes. O CPOD observado foi de 4,13 $( \pm 4,5)$. Em relação à qualidade de vida, observou-se que essa população apresentou boa qualidade de vida com OHIP de $4,9( \pm 5,6)$ sem qualquer impacto negativo relacionado à saúde bucal. Não houve associação estatisticamente significativa entre o gênero e a dentição hígida e entre biofilme visível e dentição hígida ( $p>0,05)$. Foi observada uma associação positiva entre a ausência de tratamento ortodôntico e dentição hígida $(p=0,01)$.

Tabela 1. Características demográficas e informações sobre a queixa principal da população estudada.

\begin{tabular}{|c|c|}
\hline Gênero & $N(\%)$ \\
\hline Feminino & $40(85,1)$ \\
\hline Masculino & $7(14,9)$ \\
\hline \multicolumn{2}{|c|}{ Período de graduação (Período= 6 meses) } \\
\hline $1-4$ & $29(61,6)$ \\
\hline $5-8$ & $12(25,6)$ \\
\hline Outros & $6(12,8)$ \\
\hline \multicolumn{2}{|l|}{ Local de infância } \\
\hline Rio de Janeiro - capital & $34(72,5)$ \\
\hline Demais cidades - Rio de Janeiro & $8(17,0)$ \\
\hline Cidades - Minas Gerais & $4(8,4)$ \\
\hline Cidades - Espírito Santo & $1(2,1)$ \\
\hline \multicolumn{2}{|l|}{ Problemas de saúde } \\
\hline Sim & $6(12,8)$ \\
\hline Não & $41(87,2)$ \\
\hline \multicolumn{2}{|c|}{ Percepção do graduando sobre a sua a própria saúde } \\
\hline Ruim & $1(2,1)$ \\
\hline Regular & $3(6,4)$ \\
\hline
\end{tabular}


FRANCO TPM, MOTTA VC, CANABARRO A, TANNURE PN. Perfil dos universitários de odontologia e o impacto da saúde bucal na qualidade de vida dessa população. Rev. Odontol. Univ. Cid. São Paulo 2018 jul/set 30(3) 256-264

ISSN 1983-5183

Boa

Ótima

Uso frequente de anti-inflamatório

\begin{tabular}{ll}
\hline Sim & $3(6,4)$ \\
Não & $44(93,6)$ \\
\hline Fumante & $47(100,0)$ \\
\hline Não & \\
\hline Uso de substâncias corantes & $25(53,2)$ \\
\hline Sim & $22(46,8)$ \\
Não & \\
\hline Algo incomoda nos seus dentes? & $35(74,5)$ \\
\hline Sim & $12(25,5)$ \\
Não & \\
\hline O quê? & $15(31,9)$ \\
\hline Cor dos dentes & $12(25,5)$ \\
Forma e posição & $2(4,3)$ \\
Gengiva & $6(12,8)$ \\
Outros & $12(25,5)$ \\
Não apresenta
\end{tabular}

Tabela 2. Dados sobre a história odontológica do paciente passada e atual.

\begin{tabular}{|c|c|}
\hline Tratamento ortodôntico & N (\%) \\
\hline Sim & $31(66,0)$ \\
\hline Não & $16(34,0)$ \\
\hline \multicolumn{2}{|l|}{ Foi finalizado? } \\
\hline Sim & $26(55,3)$ \\
\hline Não & $5(10,6)$ \\
\hline Outros & $16(34,0)$ \\
\hline \multicolumn{2}{|l|}{ Sensibilidade dentária } \\
\hline Sim & $3(6,4)$ \\
\hline Não & $44(93,6)$ \\
\hline \multicolumn{2}{|l|}{ Retração gengival } \\
\hline Sim & $7(14,9)$ \\
\hline Não & $40(85,1)$ \\
\hline \multicolumn{2}{|l|}{ Biofilme visível } \\
\hline Sim & $17(36,2)$ \\
\hline Não & $30(63,8)$ \\
\hline \multicolumn{2}{|l|}{ Dentes hígidos } \\
\hline Sim & $21(44,7)$ \\
\hline Não & $26(55,3)$ \\
\hline
\end{tabular}

$37(78,7)$

$6(12,8)$

$(6,4)$

)


FRANCO TPM, MOTTA VC, CANABARRO A, TANNURE PN. Perfil dos universitários de odontologia e o impacto da saúde bucal na qualidade de vida dessa população. Rev. Odontol. Univ. Cid. São Paulo 2018 jul/set 30(3) 256-264

ISSN 1983-5183

\begin{tabular}{ll}
\hline Fluorose & \\
\hline Sim & $3(6,4)$ \\
\hline Não & $44(93,6)$ \\
\hline
\end{tabular}

\section{DISCUSSÃO}

O aumento da procura pela estética do sorriso nos consultórios odontológicos fez surgir novos materiais para facilitar o trabalho do profissional e conforto do paciente. Para realização do clareamento dental de uso caseiro os materiais mais utilizados são o peróxido de carbamida para uso noturno e o peróxido de hidrogênio para uso diurno ${ }^{13}$. Todos os universitários incluídos neste estudo buscavam o clareamento caseiro e a grande maioria estava insatisfeita com algum aspecto relacionado aos dentes. A partir do interesse desses jovens em alcançar a estética desejada objetivou-se identificar o perfil e as características referentes à saúde geral e bucal dessa população e o impacto na sua qualidade de vida.

A grande maioria da amostra foi composta por jovens adultos do gênero feminino, saudáveis, não fumantes, que passaram a infância no Rio de Janeiro e que já haviam buscado o tratamento ortodôntico em algum momento da vida. Quase a metade dessa população possuía a dentição hígida (44,7\%). Esses dados mostram uma grande diferença quando comparados a um estudo prévio com universitários $(\mathrm{n}=68)$ de uma instituição pública: a grande maioria eram homens, com renda familiar de até um mil e quinhentos reais, 17,6\% fumavam e 5 usavam aparelho ortodôntico e foram excluídos da amostra ${ }^{3}$. Neste estudo prévio a amostra era composta por moradores de residências estudantis que são considerados em situação de vulnerabilidade social e econômica no Brasil devido à condição socioeconômica mais baixa. Os autores identificaram uma condição de saúde bucal desfavorável, com alta necessidade de tratamento e problemas bucais constituindo causa de absenteísmo na universidade. Vale ressaltar que, apesar da renda familiar não ter sido investigada neste estudo, sabe-se que o custo de um curso de odontologia em uma universidade particular é alto e provavelmente a população estudada apresentava uma situação financeira superior.

Sabe-se que a doença cárie é mais prevalente em grupos específicos e em populações pobres. Neste estudo o CPOD observado foi de 4,13 $(4,5)$ e a maioria apresentava dentição hígida. Mais da metade da população $(66 \%)$ tinha se submetido ao tratamento ortodôntico. Não foi observada associação estatisticamente significativa entre o gênero e a dentição hígida e entre biofilme visível e dentição hígida ( $p>0,05)$. Entretanto, observou-se uma associação positiva entre a ausência de tratamento ortodôntico e dentição hígida $(p=0,01)$, denotando uma relação entre uso de aparelho ortodôntico, acúmulo de biofilme e cárie dentária. No estudo de Freire et al. ${ }^{3}$ (2012), dos 63 estudantes examinados, apenas dez (15,9\%) estavam livres de cárie.

Por outro lado, a maloclusão e a fluorose atingem indivíduos independente da classe social. A primeira atinge em média 38\% das crianças brasileiras com 12 anos de idade ${ }^{8}$ e também pode impactar negativamente a qualidade de vida ${ }^{14}$. A fluorose, na população estudada, apresentou baixa prevalência quando comparada aos dados do Projeto SB Brasil. Vale ressaltar que nas formas mais leves de fluorose dentária, como observado neste estudo, o atrito mecânico resultante da escovação dos dentes com dentifrício pode produzir uma remissão gradativa das lesões fluoróticas através da remoção da camada mais superficial de esmalte. Os incisivos centrais superiores - os dentes mais importantes para a estética do sorriso - são especialmente susceptíveis a esses efeitos ambientais ${ }^{7}$, que podem ter acometido essa população de jovens adultos e ter contribuído, portanto, para a observação pouco frequente dessa patologia 
FRANCO TPM, MOTTA VC, CANABARRO A, TANNURE PN. Perfil dos universitários de odontologia e o impacto da saúde bucal na qualidade de vida dessa população. Rev. Odontol. Univ. Cid. São Paulo 2018 jul/set 30(3) 256-264

ISSN 1983-5183

nos universitários que compuseram a amostra.

Apesar de seu surgimento relativamente recente, a Qualidade de Vida relacionada com a Saúde Oral (Oral Health Related Quality of Life - OHRQoL) tem implicações importantes para a prática clínica e a pesquisa odontológica15. Para se avaliar a qualidade de vida, utilizou-se o questionário OHIP 14, adaptado e validado no Brasil'"1 , cujas questões são organizadas em dimensões como a limitação funcional, dor física, desconforto psicológico, incapacidade física, incapacidade psicológica, incapacidade social e limitação física. Não foi observado impacto negativo relacionado à saúde bucal na qualidade de vida dessa população. Em contrapartida, no estudo previamente relatado ${ }^{3}$ identificou-se o grau de interferência da condição bucal no desempenho diário e dificuldade para comer, incômodo para escovar os dentes, apresentar-se irritado ou nervoso, e vergonha para sorrir foram os impactos associados mais prevalentes. Os autores descreveram ainda relatos comuns de incômodo para estudar e as faltas às aulas na universidade por causa de problemas bucais $^{3}$.

O estilo de vida é a forma como um indivíduo, ou até mesmo como um grupo de pessoas vivem. Refere-se ao comportamento e escolhas feitas em relação aos hábitos do cotidiano. Dessa forma, o estilo de vida reflete diretamente no estado de saúde do indivíduo e isso tende a afetar sua percepção da QV*. Diante da população estudada, não foi observado impacto na qualidade de vida, uma vez que o valor encontrado foi de 4,9, próximo de 0 em uma escala de 0-50. Tal resultado não foi surpreendente, pois esperava-se não encontrar impacto na qualidade de vida dessa população, já que apresentava boa saúde bucal. Caso algum universitário apresentasse uma situação muito diferente do encontrado, acredita-se que teria livre acesso ao tratamento odontológico, pois o ambiente de estudo favoreceria essa situação.

Vale ressaltar aqui os limites do estudo em questão, como, por exemplo, a inclusão de uma amostra de conveniência composta por universitários de odontologia que buscavam o clareamento dentário. Ainda em relação a essa amostra, a maioria foi composta por mulheres, confirmando-se dados já conhecidos sobre o interesse maior do gênero em relação à estética e os cuidados com a saúde. A pesquisa foi realizada através de dados coletados por um questionário que tem limitações inerentes ao próprio instrumento, como, por exemplo, o viés de memória. Por outro lado, somente a partir dessa pesquisa pôde-se conhecer os universitários de odontologia da instituição pesquisada e identificar as necessidades dessa população, enfatizando, assim, a importância do cuidado e do bem-estar de jovens adultos.

\section{CONCLUSÃO}

Pode-se concluir que a maioria dos universitários se encontravam saudáveis e com a dentição hígida, entretanto, estavam insatisfeitos em relação à forma, posição e cor dos dentes. Não foi observado impacto negativo na qualidade de vida relacionada à saúde bucal. 
ISSN 1983-5183

\section{REFERÊNCIAS}

1. TSOUROS AD, Dowding G, Thompson J, Dooris M. Health promoting universities: concept, experience and framework for action. Copenhagen: World Health Organization; 1998. [Acesso em: 13 nov. 2018]; Disponível em: http://apps.who.int/iris/handle/10665/108095.

2. MELLO ALSF, Moysés ST, Moysés SJ. A universidade promotora de saúde e as mudanças na formação profissional. Interface (Botucatu) 2010 set.;14(34):683-92.

3. FREIRE MCM, Martins ÂB, Santos CR, Martins NO, Filizzola EM, Jordão LMR, et al. Condição de saúde bucal, comportamentos, autopercepção e impactos associados em estudantes universitários moradores de residências estudantis Rev odontol UNESP (Online) 2012 maio-jun.;41(3):185-91.

4. VEIGA C, Cantorani JRH, Vargas LM. Qualidade de vida e alcoolismo: um estudo em acadêmicos de licenciatura em educação física. Conexões 2016 jan.-mar.;14(1):20-34.

5. GOTTARDO AC, Webber LP, Rossa J, Arossi GA. Perda dental anterior influencia a qualidade de vida relacionada a saúde oral. Rev Iniciação Científica da ULBRA 2015 1(13):6070.

6. SISCHO L, Broder HL. Oral health-related quality of life: what, why, how, and future implications. J Dent Res 2011 Nov.;90(11):1264-70.

7. CURY JA, Tenuta LMA. Evidências para o uso de fluoretos em odontologia. Odontol baseada em evidências 2010 2(4):1-18.

8. BRASIL. Ministério da Saúde. Secretaria de Atenção à Saúde. Secretaria de Vigilância em Saúde. SB Brasil 2010: pesquisa nacional de saúde bucal: resultados principais. Brasília: Ministério da Saúde; 2012. [Acesso em: 13 nov. 2018]; Disponível em: http:// bvsms.saude.gov.br/bvs/publicacoes/pesquisa nacional saude bucal.pdf.

9. PETERSEN PE, Kandelman D, Arpin S, Ogawa H. Global oral health of older people--call for public health action. Community dental health 2010 Dec;27(4 Suppl 2):257-67.

10. STEELE JG, Sanders AE, Slade GD, Allen PF, Lahti S, Nuttall N, et al. How do age and tooth loss affect oral health impacts and quality of life? A study comparing two national samples. Community dentistry and oral epidemiology 2004 Apr;32(2):107-14.

11. OLIVEIRA BH, Nadanovsky P. Psychometric properties of the Brazilian version of the Oral Health Impact Profile-short form. Community dentistry and oral epidemiology 2005 Aug;33(4):307-14.

12. SANDERS AE, Spencer AJ, Slade GD. Evaluating the role of dental behaviour in oral health inequalities. Community dentistry and oral epidemiology 2006 Feb;34(1):71-9.

13. SILVA FMM, Nacano LG, Pizi ECG. Avaliação clínica de dois sistemas de clareamento dental. ROBRAC 2012 out.;21(56):473-9.

14. DIMBERG L, Arnrup K, Bondemark L. The impact of malocclusion on the quality of life among children and adolescents: a systematic review of quantitative studies. European journal of orthodontics 2015 Jun;37(3):238-47. 
FRANCO TPM, MOTTA VC, CANABARRO A, TANNURE PN. Perfil dos universitários de odontologia e o impacto da saúde bucal na qualidade de vida dessa população. Rev. Odontol. Univ. Cid. São Paulo 2018 jul/set 30(3) 256-264

ISSN 1983-5183

15. BENNADI D, Reddy CV. Oral health related quality of life. Journal of International Society of Preventive \& Community Dentistry 2013 Jan;3(1):1-6.

RECEBIDO EM 27/11/2017

ACEITO EM 28/09/2018 